\title{
Multiuser Detection with Base Station Diversity *
}

\author{
Matthew C. Valenti and Brian D. Woerner \\ Mobile and Portable Radio Research Group \\ Virginia Polytechnic Institute and State University \\ Blacksburg, VA 24061-0350, USA \\ email: valenti@vt.edu, phone: 540-231-0838, fax: 540-231-2968
}

\begin{abstract}
A method for combining multiuser detection with base station diversity is presented for the uplink of a multiple access network composed of $K$ transmitters and $M$ receivers. At each base station, a $K$ user soft-output multiuser receiver is implemented using the log Maximum A Posteriori (log-MAP) algorithm. The loglikelihood ratio outputs from the $M$ base stations are combined before a final hard decision is made. This strategy has the ability to simultaneously mitigate the detrimental effects of both multiuser interference and fading, and has the potential to extend cell range and reduce the frequency reuse distance. Simulation results are shown for a Time Division Multiple Access (TDMA) network with 3 users and 3 base stations.
\end{abstract}

\section{Introduction}

The performance of multiple access networks is limited by multiple access interference (MAI) and fading. The detrimental effects of MAI can be mitigated by multiuser detection (MUD) [1]. While MUD is usually considered for use with Direct Sequence Code Division Multiple Access (DS-CDMA) systems, it can also be used to improve the performance of Time Division Multiple Access (TDMA) cellular systems [2]. When used for DS-CDMA systems, the objective of MUD is to jointly detect signals that originate from the same cell (i.e. intracell interference). When used for TDMA systems, however, the objective of MUD is to jointly detect the desired signal and co-channel interferers originating from nearby cells (i.e. intercell interference).

In [2] it was shown that a noticeable performance gain can be achieved by using MUD at each base station of a TDMA system. However, as the carrier to interference ratio $(C / I)$ increases, the gain from using MUD diminishes due to the weak signal strength of the interferers. This limitation can be overcome by shar-

${ }^{*}$ This work has been supported by the Bradley Fellowship, the MPRG Industrial Affiliates Foundation, and the Office of Naval Research. ing information among base stations. For example, each base station in a TDMA network could pass the output of its MUD to the base stations that serve the strongest co-channel interferers. A second iteration of MUD could then be performed at each base station, this time incorporating the a priori information derived by nearby base stations. Performance is improved because each multiuser detector is able to exploit the decisions made by other multiuser detectors. The disadvantage of this strategy is that it requires data to be fed back from each base station to a subset of the other base stations in the network. This requires a dedicated communications link between base stations. Additionally, the iterative nature of the strategy requires that two or more passes of multiuser detection must be performed, which significantly increases the latency of the system.

A simpler approach considered in this paper is to combine the outputs of the base stations according to a diversity combining rule. If the outputs of the multiuser detectors are in the form of log-likelihood ratios (LLR's), then the outputs of the multiuser detectors can simply be added together, or "fused", according to an equal gain combining rule. This method does not require feedback and thus does not increase latency or require communications between base stations. The strategy requires that the MUD at each base station produce a log-likelihood ratio. Because the optimal multiuser detector is implemented by the Viterbi algorithm, which produces hard bit decisions, it is not suitable for such an application [1]. However, several related algorithms, such as the soft output Viterbi algorithm (SOVA) [3] and the maximum a posteriori (MAP) algorithm [4], could be used to implement the MUD such that it produces LLR's.

Diversity combining the outputs of several base stations' multiuser detectors has advantages beyond just combating MAI. Because observations from spatially separated locations are used, the negative impact of fading is reduced. If there is a deep fade between a transmitter and a particular base station, then the probability of experiencing a deep fade between the same transmitter and a different base station is very small. The LLR output corresponding to the unfaded 
path will be significantly higher than the LLR corresponding to the faded path, and thus more confidence will be placed on the output of the multiuser detector with the better path. Also, the range of the cells can be extended because the transmitted energy is received at several locations.

In this paper, we consider the application of both multiuser detection and base station diversity to TDMA cellular systems. At each base station, a soft-output multiuser detection algorithm is employed. The data produced by each of the base stations is collected at a central location and fused using equal gain combining. We investigate, through simulation, a network of three users and three base stations which models the first tier of co-channel interferers when 120 degree sectorized antennas are used. The approach of combining the outputs of multiple base stations considered here is similar to the multiplydetected macrodiversity (MDM) scheme of [5], which is a method of combining the hard bit decisions made by conventional receivers at multiple base stations. Our method differs from [5] in that a soft-output multiuser detector is employed at each base station and diversity combining uses log-likelihood ratios rather than hard bit decisions.

The remainder of this paper is organized as follows: In section II, a general model for a $K$ transmitter, $M$ receiver multiple-access network is presented. In section III, the strategy for combined multiuser detection and base station diversity reception is proposed. Section IV shows simulation results for a $K=3$ transmitter, $M=3$ receiver network over the fullyinterleaved Rayleigh flat-fading channel using TDMA signaling.

\section{System Model}

Consider a TDMA network consisting of $K$ asynchronous BPSK transmitters and $M$ independent receivers. The signal at receiver $m$ is the noisy sum of $\mathrm{K}$ delayed and attenuated signals contributed by each of the $K$ transmitters

$$
r_{m}(t)=\sum_{k=1}^{K} s_{k, m}(t)+n_{m}(t)
$$

where $n_{m}(t)$ is a complex additive white Gaussian noise (AWGN) process with variance $\sigma^{2}=N_{o} / 2$ and $s_{k, m}(t)$ is the signal at receiver $m$ due to user $k$

$$
s_{k, m}(t)=\sum_{i=1}^{L} \sqrt{P_{k, m}[i]} b_{k}[i] a_{k}\left(t-i T-\tau_{k, m}\right) e^{j \phi_{k, m}} .
$$

$L$ is the frame size (in symbols), $P_{k, m}[i]$ is the instantaneous power of user $k$ at receiver $m$ during the $i^{\text {th }}$ signaling interval, $b_{k}[i] \in\{-1,1\}$ is the $i^{\text {th }}$ symbol of user $k, a_{k}(t)$ is the signature waveform of user $k$ transmitted during the symbol interval $[0, T), \tau_{k, m}$ is the delay of user $k$ at receiver $m$ assumed to be uniformly distributed over one symbol period $T$, and $\phi_{k, m}$ is the carrier phase of user $k$ at receiver $m$ assumed to be uniformly distributed over the interval $[0,2 \pi)$. In the case of DS-CDMA, the signature sequences $\left\{a_{k}(t)\right\}$ are unique to each user, while in the case of TDMA the signature sequence is common to all users and has the form

$$
a(t)= \begin{cases}1, & \text { for } 0 \leq t<T \\ 0, & \text { elsewhere }\end{cases}
$$

For the remainder of this discussion, we will assume TDMA signaling. Without loss of generality, it is assumed that the indices of the $K$ users are assigned such that the relative delays $\tau_{k, m}$ are in ascending order, i.e. $\tau_{1, m} \leq \tau_{2, m} \leq \ldots \leq \tau_{K, m}$.

For AWGN channels, the power $P_{k, m}[i]$ is constant for all $i$. For fully interleaved flat Rayleigh fading channels, each amplitude $\sqrt{P_{k, m}[i]}$ is an independent realization of a Rayleigh random variable. Fading is assumed to be slow, and thus the fading amplitude is constant for the entire bit interval.

The received signal is passed through a bank of matched filters with output

$y_{k, m}[i]=\int_{-\infty}^{\infty} \Re\left\{r_{m}(t) a\left(t-i T-\tau_{k, m}\right) e^{-j \phi_{k, m}}\right\} d t$,

where $y_{k, m}[i]$ denotes the matched filter output at receiver $m$ corresponding to the $i^{t h}$ bit of user $k$, and $\Re\{\cdot\}$ denotes the real part. The vector $\mathbf{y}_{m}$ of matched filter outputs constitute a set of sufficient statistics for receiver $m$ and is created according to

$$
\begin{aligned}
\mathbf{y}_{m}= & {\left[y_{1, m}[1], y_{2, m}[1], \ldots, y_{K, m}[1], \ldots,\right.} \\
& \left.y_{1, m}[L], y_{2, m}[L], \ldots, y_{K, m}[L]\right]^{T} .
\end{aligned}
$$

The set of matched filter outputs from the $M$ independent receivers are collected into a matrix $\mathbf{Y}$

$$
\mathbf{Y}=\left[\mathbf{y}_{1}, \ldots, \mathbf{y}_{m}, \ldots, \mathbf{y}_{M}\right] .
$$

Similarly, a vector $\mathbf{b}$ is defined by

$$
\begin{aligned}
\mathbf{b}= & {\left[b_{1}[1], b_{2}[2], \ldots, b_{K}[1], \ldots,\right.} \\
& \left.b_{1}[L], b_{2}[L], \ldots, b_{K}[L]\right]^{T} .
\end{aligned}
$$

A vector of fading amplitudes $\mathbf{c}_{m}$ at receiver $m$ is created according to

$$
\begin{aligned}
\mathbf{c}_{m}= & {\left[\sqrt{P_{1, m}[1]}, \sqrt{P_{2, m}[1]}, \ldots, \sqrt{P_{K, m}[1]}, \ldots,\right.} \\
& \left.\sqrt{P_{1, m}[L]}, \sqrt{P_{2, m}[L]}, \ldots, \sqrt{P_{K, m}[L]}\right]^{T} .
\end{aligned}
$$


The fading amplitudes at all $M$ receivers are collected into the matrix $\mathbf{C}$

$$
\mathbf{C}=\left[\mathbf{c}_{1}, \ldots, \mathbf{c}_{m}, \ldots, \mathbf{c}_{M}\right] .
$$

Finally, a $(K-1)$ by $K$ matrix of signal cross correlations is defined for each receiver by

$$
G_{i, j}^{(m)}=\frac{\tau_{i+j, m}-\tau_{j, m}}{T} \cos \left(\phi_{i+j, m}-\phi_{j, m}\right),(10
$$

if $i+j \leq K$, and

$$
\begin{aligned}
G_{i, j}^{(m)}= & \frac{T-\left(\tau_{j, m}-\tau_{i+j-K, m}\right)}{T} \\
& \cdot \cos \left(\phi_{j, m}-\phi_{i+j-K, m}\right),
\end{aligned}
$$

if $i+j>K$. The entries of the $j^{t h}$ column of $G^{(m)}$ are the correlations (at receiver $m$ ) of the $j^{t h}$ user with the $K-1$ preceeding users.

\section{Distributed Multiuser Recep- tion}

The proposed distributed multiuser reception technique consists of a soft-output multiuser detector, or multiuser estimator, at each of the $M$ base stations followed by diversity combining of the soft-outputs.

\subsection{Multiuser Detection}

Under the assumption of equiprobable bits and Gaussian noise, the optimal multiuser detector at receiver $m$ selects the vector of estimated transmitted bits according to [1]

$$
\hat{\mathbf{b}}_{m}=\arg \left\{\max _{\mathbf{b}} \sum_{i=1}^{N} \lambda_{i, m}(\mathbf{b})\right\} .
$$

where

$$
\begin{aligned}
\lambda_{i, m}(\mathbf{b})= & 2 b_{i} c_{i, m} y_{i, m}-\left(c_{i, m}\right)^{2} \\
& -2 b_{i} c_{i, m} \sum_{j=1}^{K-1} b_{i-j} c_{i-j, m} G_{K-j, \kappa(i)}^{(m)}
\end{aligned}
$$

with $b_{i}=0$ for $i \leq 0 . \quad \kappa(i)=K$ if $K$ divides $i$, otherwise $\kappa(i)$ is $i$ modulo $K$. The estimate $\hat{\mathbf{b}}_{m}$ can be recursively computed using the Viterbi algorithm, using the branch metric given by (13).

\subsection{Multiuser Estimation}

Use of the standard Viterbi algorithm with metric given by (13) results in hard bit decisions, which are not suitable for the proposed base station diversity combining technique. Instead, a soft output in the form of a $\log$-likelihood ratio (LLR) is desired from each multiuser receiver

$$
\hat{\Lambda}_{i, m}=\log \frac{P\left(\hat{b}_{i, m}=+1 \mid \mathbf{y}_{m}\right)}{P\left(\hat{b}_{i, m}=-1 \mid \mathbf{y}_{m}\right)} .
$$

Several algorithms can be used to obtain the LLR of (14). Optimal soft-output multiuser detection can be implemented using the Maximum A Posteriori (MAP) algorithm [4], the log-MAP algorithm [6], or the Optimum Soft-Output Multiuser Estimation (OSOME) algorithm [7]. While all these algorithms offer optimal performance, they suffer from high computational complexity and large storage requirements. The log-MAP algorithm eases the computational complexity requirement by performing operations in the log-domain, while the OSOME algorithm eases storage requirements by eliminating the need for a backward recursion. Acceptable performance can be obtained by using suboptimal soft-output multiuser detection, which can be implemented using the Soft-Output Viterbi Algorithm (SOVA) [3], the max-log MAP algorithm [6], or the Suboptimum Soft-Output Multiuser Estimation (SSOME) algorithm [7]. For the purposes of this paper, we consider soft-output multiuser estimation using the log-MAP algorithm which offers excellent performance at reasonable complexity. For details on how the log-MAP algorithm can be modified for use as a multiuser detector see reference [8].

\subsection{Data Fusion}

When soft-output multiuser detection is used at each base station, performance can be improved further by using base station diversity. The LLR outputs of each receiver are passed to a central processing location where they are combined according to the following equal gain combining rule

$$
\hat{\Lambda}_{i, 0}=\sum_{m=1}^{M} \hat{\Lambda}_{i, m},
$$

After combining, a hard-decision is produced according to

$$
\hat{b}_{i, 0}= \begin{cases}+1, & \text { if } \hat{\Lambda}_{i, 0} \geq 0 \\ -1, & \text { if } \hat{\Lambda}_{i, 0}<0 .\end{cases}
$$

We also considered maximal ratio combining (MRC) in our simulations, but found that performance was consistently worse with MRC than with equal gain combining. This is as expected, because the channel state information is already incorporated into the log-MAP decoding algorithm. If the outputs of the log-MAP decoders were weighted by the channel estimates, as per maximal ratio combining, then the diversity combiner would be overcompensating and performance will suffer. 


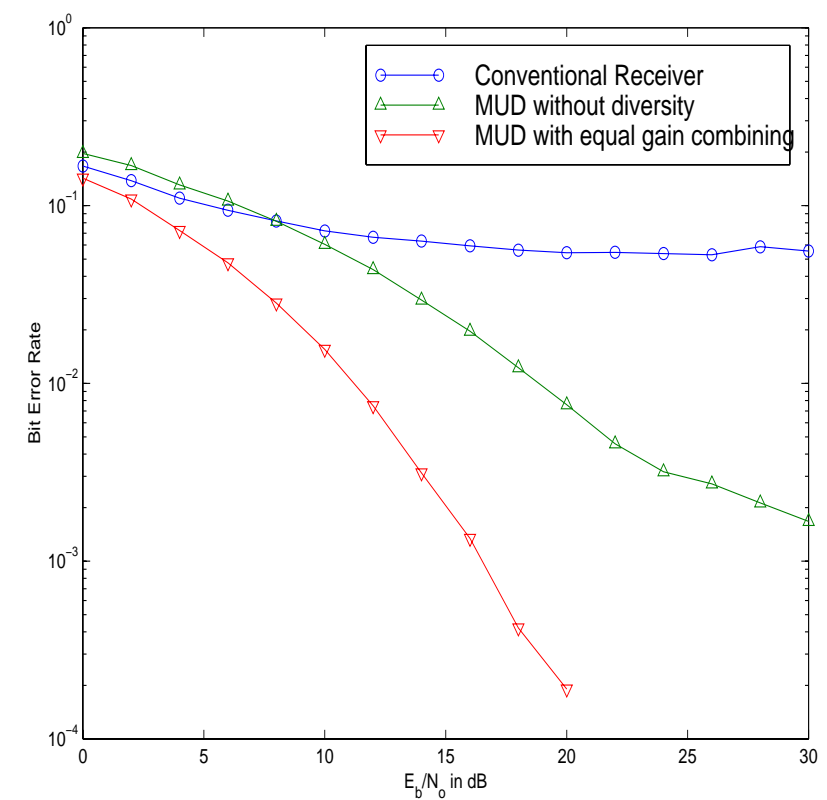

Figure 1: Bit error performance of a TDMA network with three users and three base stations in a fullyinterleaved Rayleigh flat-fading channel as parameterized by reception technique. $C / I=7 \mathrm{~dB}$ and $E_{b} / N_{o}$ ranges from 0 to $30 \mathrm{~dB}$.

\section{Simulation Results}

The uplink performance of a TDMA system using the proposed method for combining multiuser detection and base station diversity was simulated for a network of $K=3$ users and $M=3$ base stations. If it is assumed that 120 degree sectorized antennas are used at each base station, then there will be at most two interferers originating from the first tier of co-channel mobiles. Thus in the simulated scenario, each base station receives signals from the desired user and the two strong co-channel interferers from the first tier. Simulations were performed for the fully-interleaved Rayleigh flat-fading channel with block sizes of $L=$ 4, 096 bits. We assume that the symbol timing and carrier phase of each user is known at each base station so that the matrix $G^{(m)}$ can be computed. Furthermore, we assume that the fading amplitudes are known precisely so that the matrix $C$ can be reliably obtained.

In Figures 1 and 2, the bit error performance of the proposed system is shown relative to the performance of conventional reception and multiuser detection without diversity combining. The conventional receiver consists of a matched filter at each base station matched to the desired signal. In this case, multiuser detection is not used and the MAI is treated as noise. Furthermore, the conventional receiver does not use base station diversity. When multiuser detection is used without diversity, MUD is implemented by the Viterbi algorithm and the output of the MUD closest to the mobile is used to measure

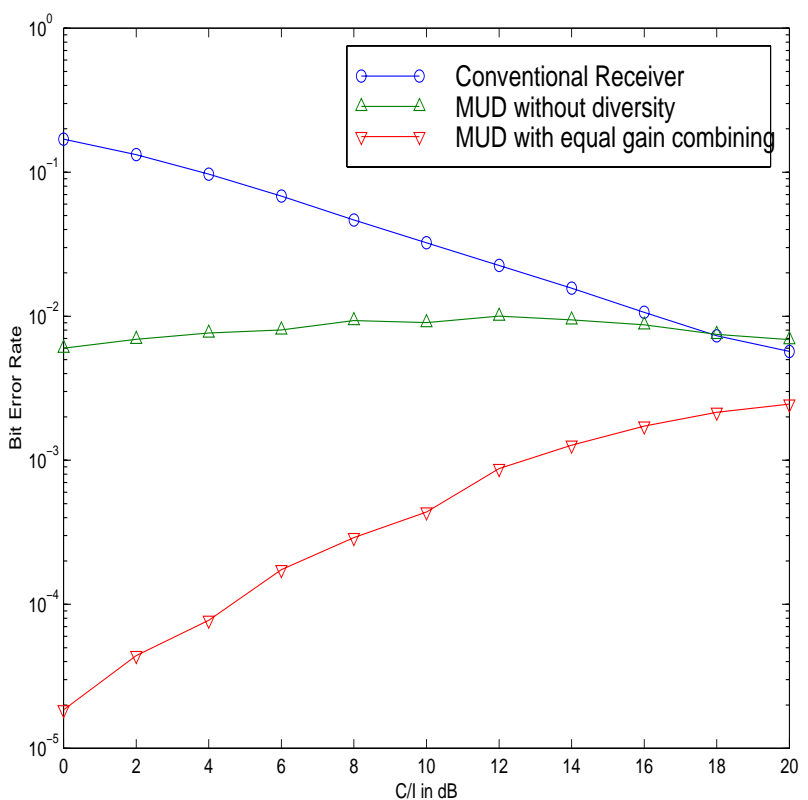

Figure 2: Bit error performance of a TDMA network with three users and three base stations in a fullyinterleaved Rayleigh flat-fading channel as parameterized by reception technique. $E_{b} / N_{o}=20 \mathrm{~dB}$ and $C / I$ ranges from 0 to $20 \mathrm{~dB}$.

performance.

The performance of this system is a function of two ratios, $E_{b} / N_{o}$ and $C / I . E_{b} / N_{o}$ is the ratio of the energy per bit to single sided noise spectral density, and quantifies the system noise. We define the carrier to interference ratio $C / I$ to be the ratio of the average power of the desired signal to the average power of each interferer. In Figure 1, the $C / I$ is set to $7 \mathrm{~dB}$, and the plot is a function of $E_{b} / N_{o}$. We chose $C / I=7 \mathrm{~dB}$ since this represents the smallest $C / I$ that a typical TDMA system will experience. In Figure $2, E_{b} / N_{o}$ is set to $20 \mathrm{~dB}$, and the plot is a function of $C / I$. For this value of $E_{b} / N_{o}$, performance is limited primarily by interference and fading, but not by noise.

From these two figures, it is apparent that significant gains can be achieved by using the proposed technique. Figure 1 shows that for $C / I=7 \mathrm{~dB}$, the performance gain increases with $E_{b} / N_{o}$. For a bit error rate (BER) of $10^{-3}$, incorporating diversity with MUD improves performance by about $15 \mathrm{~dB}$ over MUD without diversity. Note that due to MAI and fading, the conventional receiver reaches a bit error rate floor at about $5 \times 10^{-2}$.

Figure 2 shows some interesting results. As expected, the performance of the conventional system improves as the $C / I$ increases, since the interference level decreases. Due to the near-far resistance of the optimal multiuser detector, the performance of the MUD without diversity combining is relatively constant for all levels of $C / I$. However, the performance for the proposed technique actually improves as the 
$C / I$ decreases. This is due to the fact that as the level of the interference increases, the base stations can better resolve the different users. This indicates that if the interference levels can be increased, by perhaps reducing the frequency reuse distance, then system performance will improve. However, if the frequency reuse distance is reduced too far, then interference from the second tier of co-channel interferers will become nonnegligible and must be considered in the analysis.

\section{Conclusion}

In this paper, a method for combining multiuser reception and base station diversity is proposed for TDMA networks. Each of $M$ base stations performs multiuser estimation of $K$ users. The soft-outputs are passed from the base stations to a central fusion location in the form of log-likelihood ratios. The log-likelihood ratios are fused before hard decisions are made.

Simulation results were shown for the case of $M=$ 3 transmitters and $K=3$ receivers and a fullyinterleaved Rayleigh flat-fading channel. For a $C / I$ ratio of $7 \mathrm{~dB}$, the multiuser detector is able to overcome the bit error floor experienced by the conventional receiver. Furthermore, an additional gain of 15 $\mathrm{dB}$ at a BER of $10^{-3}$ is achieved when the LLR outputs of the MUD's are combined. Simulation results show that the performance of the proposed system improves as the interference level increases, raising the possibility of decreasing the frequency reuse distance.

The simulations assumed that the fading amplitudes of each user is known at each receiver, and that perfect timing and carrier synchronization is achieved for each user at each receiver. While it is possible to obtain reliable channel information for the desired user at each base station, obtaining reliable channel information for the interferers is more problematic. Thus the topic of channel estimation must be further addressed before this technique can be practically applied.

This study has shown the potential of the proposed system, but does not quantify benefits in terms of increased system capacity. A more detailed analysis should be undertaken to show the impact of using this system on the frequency reuse distance, and equivalently on the number of users supported by the system.

Finally, it is noted that this paper only discusses uncoded systems. Since all practical digital cellular systems employ error correction coding, the impact on coded performance should also be considered. Furthermore, soft-output channel decoding can be used to obtain reliability information about the user's bits, which in turn could be fed back to the multiuser detectors and used as a priori information. This approach is considered in [9].

\section{References}

[1] S. Verdu, "Minimum Probability of Error for Asynchronous Gaussian Multiple Access Channels," IEEE Trans. Info. Theory, vol. 32, no. 1, pp. 85-96, Jan. 1986.

[2] M.C. Valenti and B.D. Woerner, "Combined multiuser reception and channel decoding for TDMA cellular systems," in Proc., IEEE Veh. Tech. Conf. (VTC) (Ottawa Canada, 1998), pp. 66-70.

[3] J. Hagenauer and P. Hoeher, "A Viterbi algorithm with soft-decision outputs and its applications," in Proc., IEEE Global Telecommun. Conf. (GLOBECOM) (Dallas TX, 1989), pp. 1680-1686.

[4] L.R. Bahl, J. Cocke, F. Jeinek, and J. Raviv, "Optimal decoding of linear codes for minimizing symbol error rate," IEEE Trans. Info. Theory, vol. 20, pp. 284-287, Mar. 1974.

[5] Z.J. Haas and C.-P. Li, "The multiply-detected macrodiversity scheme for wireless cellular systems," IEEE Trans. Veh. Tech., vol. 47, pp. 506-530, May 1998.

[6] P. Robertson, P. Hoeher, and E. Villebrun, "Optimal and sub-optimal maximum a posteriori algorithms suitable for turbo decoding," European Trans. on Telecommun., vol. 8, pp. 119-125, Mar./Apr. 1997.

[7] A. Hafeez and W.E. Stark, "Soft-output multiuser estimation for asynchronous CDMA channels," in Proc., IEEE Veh. Tech. Conf. (VTC) (Phoenix AZ, 1997), pp. 465-469.

[8] M.C. Valenti and B.D. Woerner, "Iterative multiuser detection for convolutionally coded asynchronous DS-CDMA," in Proc. IEEE Int. Symp. on Personal, Indoor, and Mobile Radio Communications (PIMRC) (Boston MA, 1998).

[9] M.C. Valenti and B.D. Woerner, "Combined multiuser detection and channel decoding with base station diversity," in Proc. IEEE Global Telecommun. Conf. (GLOBECOM), Commun. Theory Mini-Conf. (Sydney Australia, November 1998), to appear. 